TRANSACTIONS OF THE

AMERICAN MATHEMATICAL SOCIETY

Volume 354, Number 12, Pages 5111-5136

S 0002-9947(02)02973-2

Article electronically published on August 1, 2002

\title{
DIFFUSIONS ON GRAPHS, POISSON PROBLEMS AND SPECTRAL GEOMETRY
}

\author{
PATRICK MCDONALD AND ROBERT MEYERS
}

\begin{abstract}
We study diffusions, variational principles and associated boundary value problems on directed graphs with natural weightings. We associate to certain subgraphs (domains) a pair of sequences, each of which is invariant under the action of the automorphism group of the underlying graph. We prove that these invariants differ by an explicit combinatorial factor given by Stirling numbers of the first and second kind. We prove that for any domain with a natural weighting, these invariants determine the eigenvalues of the Laplace operator corresponding to eigenvectors with nonzero mean. As a specific example, we investigate the relationship between our invariants and heat content asymptotics, expressing both as special values of an analog of a spectral zeta function.
\end{abstract}

\section{INTRODUCTION}

In this paper we study relationships between combinatorial analogs of various probabilistic and geometric objects associated to domains in a Riemannian manifold. To precisely state our results, we begin by establishing some notation.

Let $G=(V, E)$ be a connected oriented bidirected graph with vertex set $V$ and edge set $E$. Given $e \in E$, we will represent $e$ as an ordered pair $e=(t(e), h(e))$, where $t(e), h(e) \in V$ (see section 2 for definitions).

We will denote by $C^{0}(G)$ the vector space of real-valued functions on $V$ and by $C^{1}(G)$ the vector space of real-valued functions on $E$. There is a natural coboundary operator $d: C^{0}(G) \rightarrow C^{1}(G)$ defined by

$$
d f(e)=f(h(e))-f(t(e)) .
$$

Let $C_{0}^{0}(G) \subset C^{0}(G)$ and $C_{0}^{1}(G) \subset C^{1}(G)$ be the subspaces consisting of those functions with compact support.

Let $W_{V}: V \rightarrow \mathbb{R}^{+}$be a vertex weighting. Associated to $W_{V}$ there is an inner product on $C_{0}^{0}(G)$ defined by

$$
\langle f, g\rangle_{V}=\sum_{x \in V} f(x) g(x) W_{V}(x) .
$$

Similarly, a function $W_{E}: E \rightarrow \mathbb{R}^{+}$defines an inner product $\langle\cdot, \cdot\rangle_{E}$ on $C_{0}^{1}(G)$.

Received by the editors July 1, 2001 and, in revised form, October 26, 2001.

2000 Mathematics Subject Classification. Primary 58J65, 58J50.

Key words and phrases. Poisson problem, random walk, variational principles, spectral graph theory, Stirling numbers, zeta functions. 
Given a pair of functions $W_{V}$ and $W_{E}$ as above, we call the ordered pair $W=$ $\left(W_{V}, W_{E}\right)$ a weighting for $G$ if

$$
W_{E}(x, y) W_{V}(x)=W_{E}(y, x) W_{V}(y),
$$

where $x, y \in V$. If $\mathcal{O}$ is an orientation for $G$ (cf. section 2) and $W$ is a weighting, we call the triple $(G, \mathcal{O}, W)$ a graph with geometry.

Given a weighting $W$, we will denote by $d_{W}^{*}: C^{1}(G) \rightarrow C^{0}(G)$ the associated adjoint of the coboundary map $d$. We will denote by $\Delta: C^{0}(G) \rightarrow C^{0}(G)$ the (vertex) Laplacian, $\Delta=d_{W}^{*} d$ (we will not investigate the edge Laplacian $d d_{W}^{*}$ ).

Interest in applications involving discrete Laplace operators of the type defined above can be traced to Kirchhoff [ $\mathrm{K}$, who modelled simple circuits as finite graphs with each edge corresponding to the conductance of a given circuit component (cf. DSn] for a survey of random walks and electrical networks). As Kirchhoff established, it is possible to give graph theoretic formulations for the laws governing current behavior in a simple circuit (Kirchhoff's laws for voltage and current, Ohm's law). Given a simple circuit (i.e., a graph with each edge weighted to represent the conductance of a given component and each vertex of weight one) and a given input current, it is possible to formulate the problem of finding the induced circuit current as a Dirichlet problem involving the edge Laplacian. In addition, there is a solution to this Dirichlet problem given by "energy minimization" (Thomson's Principle, cf. [B] $)$.

Since Kirchhoff, the study of graph Laplacians has yielded a remarkable wealth of information in a variety of contexts (the references $[\mathrm{B},[\mathrm{C}]$ and references therein provide expository introductions to some of these applications). Among those fields where graphs and their associated discrete boundary value problems have found interesting applications are potential theory (cf. [B], [Du, and references therein), spectral theory (cf. [DS], Ge, [C]), and differential geometry and global analysis (cf. [Do], [F1, [F2], [V1], [V2]). In this paper, we focus on several such applications. To be more precise, let $W=\left(W_{V}, W_{E}\right)$ be a weighting for $G$ and define the associated vertex weighting as

$$
w_{V}(x)=\sum_{(x, y) \in E} W_{E}(x, y)
$$

Let

$$
p(x, y)=\frac{W_{E}(x, y)}{w_{V}(x)}
$$

be the transition probabilities for a random walk, denoted $X_{n}$, on the vertices of $G$. For $x \in V$, let $P^{x}$ be the associated measure charging trajectories beginning at $x$ and let $E^{x}$ be the corresponding expectation operator (a classical reference for random walks is $[\mathrm{Sp} ; \mathrm{A}]$ is a survey concerning the role of random walks in a variety of contexts). Let $D \subset G$ be a domain of $G$ (cf. Definition 2.2) and let $\eta$ be the index of the first exit time from the interior of $D$ (cf. Definition 2.4):

$$
\eta=\inf \left\{n \geq 0: X_{n} \notin D\right\} .
$$

Rescaling transition times to reflect graph geometry, let $\tau$, the first exit time from the interior of $D$, be defined by (cf. Definition 2.4)

$$
\tau=\sum_{n=0}^{\eta-1} \frac{1}{w_{V}\left(X_{n}\right)} .
$$


Many of the applications of the study of graph Laplacians to geometry and analysis involve the relationship of exit times and hitting times for random walks on $G$ to the discrete Laplace operator and a corresponding theory of boundary value problems. Our first result is a consequence of identifying the precise relationship between exit time moments of the natural random walk described above and solutions to natural Poisson problems involving graph Laplacians on $G$ (cf. Theorem 4.1). More precisely, given a domain $D$ of $G$ and a positive integer $k$, define real numbers $A_{1, k}=A_{1, k}(D)$ and $A_{2, k}=A_{2, k}(D)$ by

$$
\begin{aligned}
& A_{1, k}=\left\langle E^{x}\left[\tau^{k}\right], 1\right\rangle_{V}, \\
& A_{2, k}=k ! \sup _{f \in C^{0}(i D) \backslash\{0\}} \frac{\langle f, 1\rangle_{i D}^{2}}{\left|\left\langle f, \Delta_{i D}^{k} f\right\rangle_{i D}\right|},
\end{aligned}
$$

where $i D$ is the collection of interior vertices of $D$ (cf. Definition 2.2), $\Delta_{i D}$ is the interior Laplace operator (cf. (2.9) and Proposition 2.3) and the inner product in (1.6) is induced by restriction to functions supported on $i D$ (cf. section 2). For reasons that will soon become apparent, we will refer to the sequence $\left\{A_{1, k}(D)\right\}$ as the moment spectrum of $D$, and we will write $\operatorname{mspec}(D)=\left\{A_{1, k}(D)\right\}$. Similarly, we will refer to the sequence $\left\{A_{2, k}(D)\right\}$ as the Poisson spectrum of $D$, and we will write $\operatorname{pspec}(D)=\left\{A_{2, k}(D)\right\}$. We prove the following fact.

Theorem 1.1. Suppose that $(G, \mathcal{O}, W)$ is a connected graph with geometry (cf. Definition 2.1). Let $p: V \times V \rightarrow \mathbb{R}$ be the transition probabilities associated to the weighting $W$ as defined in (1.3) above, and let $X_{n}$ be the random walk determined by the transition probabilities $p(x, y)$. Let $D$ be an $\alpha$-weight regular domain (cf. Definition (2.5)). For $k$ a positive integer, let $A_{1, k}$ and $A_{2, k}$ be defined by (1.5) and (1.6), respectively. Then

$$
A_{1, k}=A_{2, k}+\sum_{j=1}^{k-1}(-\alpha)^{j-k}\left\{\begin{array}{l}
k \\
j
\end{array}\right\} A_{2, j}
$$

where the coefficients $\left\{\begin{array}{l}k \\ j\end{array}\right\}$ are the Stirling numbers of the second kind (cf. Definition (4.1)). Similarly,

$$
A_{2, k}=A_{1, k}+\sum_{j=1}^{k-1}(-\alpha)^{j-k}\left[\begin{array}{l}
k \\
j
\end{array}\right] A_{1, j}
$$

where the coefficients $\left[\begin{array}{l}k \\ j\end{array}\right]$ are the Stirling numbers of the first kind (cf. Definition (4.1)).

Theorem 1.1 implies that for every weight regular domain $D, \operatorname{mspec}(D)$ determines $\operatorname{pspec}(D)$ and vice versa.

Theorem 1.1 is motivated in part by an analogous result concerning Brownian motion on smoothly bounded domains in Riemannian manifolds. The precise statement is as follows: Let $M$ be a manifold with Riemannian metric $g$. Suppose that $X_{t}$ is classical Brownian motion in $M$, that $D \subset M$ is a smoothly bounded domain with compact closure, and that $\tau$ is the first exit time of Brownian motion from $D$. Let $P^{x}$ be the measure charging paths beginning at $x \in M$, and for $k$ a positive 
integer, let $B_{1, k}$ be defined by

$$
B_{1, k}=\int_{D} E^{x}\left[\tau^{k}\right] d g
$$

where $E^{x}$ is expectation with respect to $P^{x}$ and $d g$ is the metric density. As a function of the domain, the sequence $\left\{B_{1, k}(D)\right\}$ has a number of interesting properties. For domains in the plane, $B_{1,1}(D)$ is known as the torsional rigidity associated to a beam of uniform cross-section $D$, and arises in the theory of elasticity. In general, the sequence $\left\{B_{1, k}(D)\right\}$ is invariant under the action of the isometry group of $M$ and hence contains geometric information for the domain $D$ (see [KMM], [M] for related work). Define

$$
B_{2, k}=k ! \sup _{f \in \mathcal{F}_{k}} \frac{\left(\int_{D} f d g\right)^{2}}{\left|\int_{D} f \mathcal{L}^{k} f d g\right|},
$$

where $\mathcal{L}$ is one half the Laplace operator and $\mathcal{F}_{k}=\left\{f \in C^{\infty}(D): \Delta^{j} f=\right.$ 0 on $\partial D, 0 \leq j<k\}$. Then for all positive integers $k$ (cf. [KM] for the Euclidean case, $[\mathrm{M}]$ for the general case)

$$
B_{1, k}=B_{2, k}
$$

Suppose we are given a Riemannian manifold $M$. A triangulation of $M$ leads to a bidirected graph with orientation, as well as approximations of the associated Laplace operator (by finite difference operators) and the Brownian motion $X_{t}$ (by random walks). These approximations give rise to analogs $\left(A_{1, k}\right)$ of the geometric invariants $B_{1, k}$, and similarly for the variational quotients. Theorem 1.1 precisely quantifies the difference which arises in approximating two geometric invariants in terms of the Stirling cycle numbers, combinatorial objects which arise naturally in a variety of contexts in graph theory and statistical mechanics.

The remainder of our results demonstrate that the invariants $A_{1, k}$ and $A_{2, k}$ play an important role in the analysis and geometry of infinite graphs. These results provide a relationship between the spectrum of the interior Laplace operator associated to a domain, the Poisson spectrum of the domain, and the moment spectrum of the domain. We begin with a definition:

Definition 1.1. Let $(G, \mathcal{O}, W)$ be a graph with geometry and let $D$ be a domain in $G$. Let $\operatorname{spec}(D)$ be the spectrum of the interior Laplace operator associated to $D$. We define the set $\operatorname{spec}^{*}(D)$ by

$$
\operatorname{spec}^{*}(D)=\left\{\lambda \in \operatorname{spec}(D):\left\langle\phi_{\lambda}, \mathbf{1}_{i D}\right\rangle_{V} \neq 0\right\},
$$

where $\phi_{\lambda}$ is a normalized eigenvector associated to $\lambda$ and $\mathbf{1}_{i D}$ is the indicator function of $i D$.

We emphasize that $\operatorname{spec}^{*}(D)$ contains no information concerning spectral multiplicity; it is a subset of the real numbers consisting of those eigenvalues whose corresponding eigenspace projects nontrivially onto constant functions.

Our second result can now be concisely stated:

Theorem 1.2. Suppose that $(G, \mathcal{O}, W)$ is a graph with geometry and that $D$ and $D^{\prime}$ are domains in $G$. Then, with the notation as in Definition 1.1,

$$
\operatorname{pspec}(D)=\operatorname{pspec}\left(D^{\prime}\right) \text { implies } \operatorname{spec}^{*}(D)=\operatorname{spec}^{*}\left(D^{\prime}\right),
$$


and we say that $\operatorname{pspec}(D)$ determines $\operatorname{spec}^{*}(D)$. Moreover, suppose $\mathcal{D}_{N}$ is the collection of all domains for which the cardinality of $\operatorname{spec}^{*}(D)$ is $N$ :

$$
\mathcal{D}_{N}=\left\{D \subset G: D \text { a domain, } \operatorname{card}\left(\operatorname{spec}^{*}(D)\right)=N\right\} .
$$

Then there are $N$ rational functions $f_{i}, 1 \leq i \leq N, f_{i}: \mathbb{R}^{2 N} \rightarrow \mathbb{R}$, such that for every $D \in \mathcal{D}_{N}$, the roots of the polynomial

$$
P_{N}(x)=x^{N}+\sum_{i=0}^{N-1} f_{i}\left(A_{2,0}(D), A_{2,1}(D), \ldots, A_{2,2 N-1}(D)\right) x^{i}
$$

give the elements of $\operatorname{spec}^{*}(D)$. When the domains under consideration are $\alpha$-weight regular, the same claim holds with $\operatorname{mspec}(D)$ replacing $\operatorname{pspec}(D)$.

Thus, given a domain $D$, any invariant defined in terms of $\operatorname{spec}^{*}(D)$ can be defined in terms of $\operatorname{pspec}(D)$ (and/or mspec $(D)$ ). An important example of such invariants are given by the notion of heat content. Recall, if $D$ is a smoothly bounded domain in a Riemannian manifold $M$ and $H(x, t)$ solves the heat equation with intial data

$$
\begin{aligned}
\Delta H & =\partial_{t} H \text { on } D \times(0, \infty), \\
H(x, 0) & =1 \text { on } D, \\
H(y, t) & =0 \text { on } \partial D \times(0, \infty),
\end{aligned}
$$

then the heat content of $D$ is the function $Q(t)$ defined by

$$
Q(t)=\int_{D} H(x, t) d g,
$$

where $d g$ is the metric density. By a theorem of van den Berg and Gilkey [BG], $Q(t)$ admits an asymptotic expansion for small $t$ :

$$
Q(t) \simeq \sum_{n=0}^{\infty} q_{n} t^{n}
$$

where the coefficients $q_{n}$ are given as integrals of metric invariants associated to $D$. The coefficients in (1.17) are sometimes referred to as the heat content asymptotics. We prove

Theorem 1.3. Suppose that $(G, \mathcal{O}, W)$ is a graph with geometry and that $D$ is a domain of $G$. Suppose the heat content asymptotics of $D$ are given by $\left\{q_{n}\right\}$ (cf. Definition (5.2)). Then $\left\{q_{n}\right\}$ determines $\operatorname{spec}^{*}(D)$. In addition, $\left\{q_{n}\right\}$ is determined by $\operatorname{pspec}(D)$, and if $D$ is $\alpha$-weight regular, the same is true with $\operatorname{pspec}(D)$ replaced by $\operatorname{mspec}(D)$.

We note that there are natural questions arising from our results. For example, to what extent can we establish converse statements for the results of Theorem 1.2 and Theorem 1.3 (i.e., to what extent does $\operatorname{spec}^{*}(D)$ determine $\operatorname{pspec}(D)$ )? We address these issues in Corollary 5.1 and Corollary 5.3, respectively, where we prove that $\operatorname{spec}^{*}(D)$ together with spectral information related to the volume of $D$ (cf. Definition 5.1) determines the Poisson spectrum, and similarly for the heat content asymptotics. In Definition 5.3 we define a variant of the spectral zeta function for the Dirichlet Laplacian. Our final result, Corollary 5.4, establishes that special values of our zeta function give both the invariants defined in (1.6) (values at positive integers) and the heat content asymptotics (values at negative integers). 
To prove our theorems, we study a natural family of Poisson problems on the domain $D$ and develop the potential theory required to analyze such systems. Of particular importance is the precise relationship between the geometry of the underlying graph, the transition operator, the inverse of the Laplace operator, and exit time moments for the natural random walk on the graph (cf. Corollary (2.1), Lemma (4.2) and Theorem (4.1)). To prove Theorem 1.1 we prove that the variational quotients appearing in (1.6) are given by integrating the solutions of the Poisson problems arising in our study (cf. Theorem 3.2), we establish an analog of Dynkin's formula (cf. Theorem 3.1), and we use this to relate the moments appearing in (1.5) to the solutions of our Poisson problems (cf. Theorem 4.1). To prove Theorems 1.2 and 1.3 we rely on the solution of the Stieltjes Moment Problem and explicit computations. Our computations provide algebraic descriptions of the invariants of interest and indicate the precise nature of the relationship between the sets of invariants.

The paper is organized as follows: In the second section we review the necessary machinery of probability, analysis and graph theory. In the third section we examine iterated solutions of discrete Poisson problems. In the fourth section we present the proof of Theorem 1.1. In the fifth and final section we present the proofs of Theorem 1.2 and Theorem 1.3, as well as a number of corollaries involving converse results.

\section{BACKGRound AND Notation}

Let $G=(V, E)$ be a connected bidirected graph with vertex set $V$ and edge set $E \subset V \times V$. Given $e \in E$, we will represent $e$ as an ordered pair $e=(t(e), h(e))$, where $t(e), h(e) \in V(h(e)$ is the terminal point of $e$ and $t(e)$ is the initial point of $e)$. We will say that a vertex $x$ is incident with an edge $e$ if $x=t(e)$ or $x=h(e)$. For $x$ a vertex, we denote by $\operatorname{deg}(x)$ the degree of $x: \operatorname{deg}(x)=$ number of $e$ such that $x$ is incident with $e$. We will restrict our attention to graphs which admit no self edges (i.e., edges of the form $(x, x)$ ). We will denote the statement " $x$ is adjacent to $y$ " by $x \sim y$.

Given an edge $e=(x, y)$, we will denote the opposite edge $(y, x)$ by $e^{-1}$. An orientation of a bidirected graph $G$ is a subset $\mathcal{O} \subset E$ satisfying $e \in \mathcal{O}$ if and only if $e^{-1} \notin \mathcal{O}$.

A vertex weighting (or volume) on a bidirected graph $G=(V, E)$ is a function $W_{V}: V \rightarrow \mathbb{R}^{+}$. An edge weighting on a bidirected graph $G=(V, E)$ is function $W_{E}: V \times V \rightarrow \mathbb{R}^{\geq 0}$ which is supported on $E$.

Definition 2.1. Suppose that $G$ is a connected bidirected graph with orientation $\mathcal{O}$, vertex weight $W_{V}$, and edge weight $W_{E}$. We say that the pair $W=\left(W_{V}, W_{E}\right)$ is a weighting for $G$ if

$$
W_{E}(x, y) W_{V}(x)=W_{E}(y, x) W_{V}(y)
$$

for all $x, y \in V$. Given a weighting $W$, we associate to $G$ a second vertex weighting, $w_{V}(x)$, called the natural auxiliary weighting, defined by

$$
w_{V}(x)=\sum_{(x, y) \in E} W_{E}(x, y)
$$

A triple $(G, \mathcal{O}, W)$, where $G$ is a connected bidirected graph, $\mathcal{O}$ is an orientation of $G$ and $W$ is a weighting for $G$, is called a graph with geometry. 
As in the introduction, we will denote by $C^{0}(G)$ the vector space of real-valued functions on $V$ and by $C^{1}(G)$ the vector space of real-valued functions on $E$. There is a natural coboundary operator $d: C^{0}(G) \rightarrow C^{1}(G)$ defined by

$$
d f(e)=f(h(e))-f(t(e)) .
$$

Let $C_{0}^{0}(G) \subset C^{0}(G)$ and $C_{0}^{1}(G) \subset C^{1}(G)$ be the subspaces consisting of those functions with compact support.

Let $(G, \mathcal{O}, W)$ be a graph with geometry. The weighting $W$ gives rise to a pair of inner products

$$
\begin{aligned}
& \langle\cdot, \cdot\rangle_{V}: C_{0}^{0}(G) \times C_{0}^{0}(G) \rightarrow \mathbb{R} \\
& \langle\cdot, \cdot\rangle_{E}: C_{0}^{1}(G) \times C_{0}^{1}(G) \rightarrow \mathbb{R}
\end{aligned}
$$

defined by

$$
\begin{aligned}
\langle f, g\rangle_{V} & =\sum_{x \in V} f(x) g(x) W_{V}(x) \\
\langle F, G\rangle_{E} & =\sum_{x \in V} \sum_{(x, y) \in \mathcal{O}} F(x, y) G(x, y) W_{E}(x, y) W_{V}(x) .
\end{aligned}
$$

The inner products associated to $W$ give rise to an adjoint map

$$
d_{W}^{*}: C_{0}^{1}(G) \rightarrow C_{0}^{0}(G),
$$

which in turn gives rise to the (vertex) Laplace operator $\Delta: C_{0}^{0}(G) \rightarrow C_{0}^{0}(G)$ defined by

$$
\Delta=d_{W}^{*} d
$$

Example 2.1. Let $V=\mathbf{Z}^{n}$ be the integer lattice in $\mathbb{R}^{n}$ (with the standard Riemannian structure). Define an edge structure by setting

$$
E=\{(x, y): x, y \in V, \operatorname{dist}(x, y) \leq 1\}
$$

where dist $(x, y)$ is the standard Euclidean distance between $x$ and $y$. The orientation of $\mathbb{R}^{n}$ induces an orientation on the graph $G=(V, E)$ :

$$
(x, y) \in \mathcal{O} \text { if the tangent to the line from } x \text { to } y \text { is positively oriented. }
$$

Define an edge weighting by

$$
W_{E}(x, y)= \begin{cases}\frac{1}{(\operatorname{dist}(x, y))^{2}} & \text { if }(x, y) \in E \\ 0 & \text { elsewhere }\end{cases}
$$

Define a vertex weighting by

$$
W_{V}(x)=1 .
$$

Then $W=\left(W_{V}, W_{E}\right)$ is a natural weighting. The corresponding Laplace operator is the standard discretization of the Laplace operator acting on functions on $\mathbb{R}^{n}$.

To generalize the above, suppose that $M$ is an oriented complete Riemannian manifold with metric $g$ and injectivity radius bounded below by a positive constant $b$. Choose a discrete collection of points $\left\{x_{i}\right\}=V \subset M$ satisfying

1. $\operatorname{dist}_{g}\left(x_{i}, x_{j}\right) \geq \frac{b}{4}$ for $i \neq j$,

2. for every $x \in M$ there is some $i$ such that $\operatorname{dist}_{g}\left(x, x_{i}\right)<\frac{b}{2}$, 
where dist $_{g}$ is the $g$-induced distance function on $M$. Define an edge structure on $V$ by the relationship

$$
x_{i} \sim x_{j} \text { if } \operatorname{dist}_{g}\left(x_{i}, x_{j}\right)<b \text { and } i \neq j .
$$

Then $G=(V, E)$ is a connected bidirected graph. The orientation on $M$ induces an orientation on $G$ : Given an edge $(x, y)$ we say $(x, y) \in \mathcal{O}$ if the tangent vector at $x$ to a distance minimizing geodesic connecting $x$ to $y$ is given by the orientation on $M$. We define an edge weighting on $E$ and a vertex weighting on $V$ by

$$
\begin{aligned}
W_{E}(x, y) & =\frac{1}{\left(\operatorname{dist}_{g}(x, y)\right)^{2}}, \\
W_{V}(x) & =1 .
\end{aligned}
$$

From the definition, it is clear that $W=\left(W_{V}, W_{E}\right)$ is a weighting and that the triple $(G, \mathcal{O}, W)$ is a graph with geometry. In the case of Euclidean space $\mathbb{R}^{n}$, taking $V=Z^{n}$, where $\mathbf{Z}^{n}$ is the integer lattice and $b=\sqrt{2}$, results in the triple being the standard nearest-neighbor graph on the given lattice, where each vertex and each edge is assigned the same weight. The associated Laplace operator is the standard finite difference operator.

In [V1] Varopoulos constructs a similar transition operator and proves that as the mesh described above becomes fine, the natural random walk converges to classical Brownian motion on $M$, and uses this fact to relate transience of Brownian motion on regular coverings of a compact Riemannian manifold to transient properties of the corresponding group of deck transformations. In addition, Varopoulos uses the random walk to give large time bounds on the heat kernel of a noncompact manifold.

Our first proposition characterizes the action of the Laplacian as a weighted average (cf. Do for the case in which each vertex has the same degree and the edge weights are constant)

Proposition 2.1. Suppose that $(G, \mathcal{O}, W)$ is a graph with geometry, $\Delta$ the associated Laplace operator. The action of $\Delta$ on a function $f$ at a vertex $x$ is given by

$$
-\Delta f(x)=\sum_{y \in V} f(y) W_{E}(x, y)-w_{V}(x) f(x),
$$

where $W_{E}$ is the edge weight function and $w_{V}$ is the auxiliary vertex weight function $(2.2)$.

Proof. For $x \in V$, let $\delta_{x} \in C_{0}^{0}(G)$ be defined by

$$
\delta_{x}(y)= \begin{cases}1, & y=x \\ 0, & \text { elsewhere }\end{cases}
$$

From the definition of the inner product (2.3),

$$
\left\langle\Delta f, \delta_{x}\right\rangle_{V}=\Delta f(x) W_{V}(x) .
$$


As $d_{W}^{*}$ is the adjoint of the coboundary operator with respect to $W$, we have, via $(2.4)$,

$$
\begin{aligned}
\left\langle\Delta f, \delta_{x}\right\rangle_{V}= & \left\langle d f, d \delta_{x}\right\rangle_{E} \\
= & \sum_{(x, y) \in \mathcal{O}}(f(y)-f(x))(-1) W_{E}(x, y) W_{V}(x) \\
& +\sum_{(y, x) \in \mathcal{O}}(f(x)-f(y)) W_{E}(y, x) W_{V}(y) .
\end{aligned}
$$

Using the fact that $\mathcal{O}$ is an orientation and relationship (2.1), we obtain

$$
\left\langle\Delta f, \delta_{x}\right\rangle_{V}=\sum_{(x, y) \in E}(f(x)-f(y)) W_{E}(x, y) W_{V}(x) .
$$

Using the definition of the auxiliary vertex weighting (2.2) and (2.7), the proof of the proposition follows.

We next develop the machinery of boundary value problems for graphs and subgraphs.

Suppose that $G=(V, E)$ is a graph and $(G, \mathcal{O}, W)$ a graph with geometry. If $A \subset V$, we define

$$
C^{0}(G, A)=\left\{f \in C^{0}(G):\left.f\right|_{V \backslash A}=0\right\} .
$$

There are natural isomorphisms $J_{A}: C^{0}(A) \rightarrow C^{0}(G, A)$ which induce inclusions

$$
I_{A}: C^{0}(A) \rightarrow C^{0}(G)
$$

and projections

$$
P_{A}: C^{0}(G) \rightarrow C^{0}(A) .
$$

The vertex weighting $W_{V}$ induces a weighting on $A$ and an inner product on compactly supported functions:

$$
\langle f, g\rangle_{A}=\left\langle I_{A} f, I_{A} g\right\rangle_{V} .
$$

Definition 2.2. Suppose that $G=(V, E)$ is a bidirected graph. Suppose that $D=\left(V^{\prime}, E^{\prime}\right)$ is a bidirected subgraph of $G$. We say that $x \in V^{\prime}$ is an interior vertex of $D$ if, for all $y \in V$ with $(x, y)$ an edge of $G$, we have that $y \in V^{\prime}$ and $(x, y) \in E^{\prime}$. We denote the collection of all interior vertices of $D$ by $i D$. We call all vertices of $D$ which are not interior vertices of $D$ boundary vertices of $D$. We denote the collection of all boundary vertices of $D$ by $\partial D$. A domain of $G$ is a finite connected bidirected subgraph of $G$ with nonempty interior vertex set.

Note that if $(G, \mathcal{O}, W)$ is a graph with geometry and $D$ is a domain of $G$, then $\mathcal{O}$ induces (by restriction) an orientation $\mathcal{O}^{\prime}$ of $D$, and $W$ induces (by restriction) a weighting $W^{\prime}$ for $D$ which coincides with $W$ at all interior vertices. The triple $\left(D, \mathcal{O}^{\prime}, W^{\prime}\right)$ gives rise to a Laplace operator $\Delta_{D}: C^{0}(D) \rightarrow C^{0}(D)$, the induced Laplace operator associated to $D$.

Proposition 2.2. Suppose that $(G, \mathcal{O}, W)$ is a graph with geometry, $\Delta$ the associated Laplace operator. Let $D$ be a domain in $G, \Delta_{D}$ the induced Laplace operator associated to $D$. Then for all $f \in C^{0}(D)$, for every interior vertex $x$ of $D$,

$$
\Delta_{D} f(x)=\Delta I_{D} f(x),
$$

where $I_{D}$ is the natural inclusion, $I_{D}: C^{0}(D) \rightarrow C^{0}(G)$. 
Proof. If $D=\left(V^{\prime}, E^{\prime}\right)$, and $\left(D, \mathcal{O}^{\prime}, W^{\prime}\right)$ denotes the domain with induced structure, then, following the proof of Proposition (2.1), we have

$$
\left\langle\Delta_{D} f, \delta_{x}\right\rangle_{V^{\prime}}=\sum_{(x, y) \in E^{\prime}}\left(I_{D} f(x)-I_{D} f(y)\right) W_{E^{\prime}}(x, y) W_{V^{\prime}}(x) .
$$

Since $W_{V}(x)=W_{V}^{\prime}(x)$ and $W_{E}(x, y)=W_{E}^{\prime}(x, y)$ at all interior vertices, (2.8) follows.

Note that it is not necessarily the case that (2.8) holds at boundary vertices: the respective auxiliary weightings at a boundary vertex may not coincide. Nonetheless, we can give a concise description of the action of the domain Laplacian as follows: enumerate the vertices of $D$ with the first $m$ vertices interior, the remaining $N-m$ vertices boundary. For $x \in D$, let $\delta_{x}$ be as given in (2.6). Then $\left\{\delta_{x_{i}}\right\}_{i=1}^{N}$ is a basis for $C^{0}(D)$ and the domain Laplacian with respect to this basis has the form

$$
\Delta_{D}=\left(\begin{array}{cc}
\Delta_{i D, i D} & \Delta_{\partial D, i D} \\
\Delta_{i D, \partial D} & \Delta_{\partial D, \partial D}
\end{array}\right),
$$

where the action of the operators $\Delta_{A, B}$ is given by

$$
\Delta_{A, B}=P_{B} \Delta_{D} I_{A},
$$

where $I_{A}$ and $P_{B}$ are the natural inclusion and projection maps. Of particular interest is the interior Laplace operator acting on functions on $i D, \Delta_{i D, i D}: C^{0}(i D) \rightarrow$ $C^{0}(i D)$. For notational ease, we will write $\Delta_{i D, i D}=\Delta_{i D}$.

Proposition 2.3. Suppose that $(G, \mathcal{O}, W)$ is a graph with geometry and that $D$ is a domain of $G$. Suppose that $\Delta_{i D}$ is the interior Laplace operator defined by (2.9) with $A=B=i D$. Then, for all $f \in C^{0}(i D)$, for all $x \in i D$,

$$
-\Delta_{i D} f(x)=\sum_{y \in i D} f(y) W_{E}(x, y)-w_{V}(x) f(x),
$$

and the interior Laplace operator is selfadjoint.

Proof. Let $x \in i D$. By definition, $\Delta_{i D} f(x)=P_{i D} \Delta_{D} I_{i D} f(x)$. By Proposition 2.2 and Proposition 2.1,

$$
\begin{aligned}
-\Delta_{D} I_{i D} f(x) & =-\Delta I_{D} f(x) \\
& =\sum_{y \in V} I_{D} f(y) W_{E}(x, y)-w_{V}(x) I_{D} f(x) .
\end{aligned}
$$

Since $I_{D} f$ is supported on $i D,(2.10)$ follows. That $\Delta_{i D}$ is selfadjoint on $C^{0}(i D)$ follows immediately from (2.10).

To study properties of the Laplace operators defined above, we develop two natural random walks. It is expression (2.5) and the corresponding variants which link the behavior of the random walks to solutions of boundary value problems involving the associated Laplace operators.

Definition 2.3. Let $(G, \mathcal{O}, W)$ be a graph with geometry. The transition probabilities associated to $(G, \mathcal{O}, W)$ are given by the function $p: V \times V \rightarrow \mathbb{R}$ defined by

$$
p(x, y)=\frac{W_{E}(x, y)}{w_{V}(x)} .
$$


Note that for every vertex $x$, the ratio $p(x, y)=\frac{W_{E}(x, y)}{w_{V}(x)}$ is a probability distribution on the collection of vertices adjacent to $x$. These transition probabilities induce a natural random walk $X_{n}$ taking values in the vertices of $G$. Let $P^{x}, x \in V$, be the associated family of probability measures charging paths beginning at $x$ and let $E^{x}$ denote the corresponding expectations.

Because $G$ is connected and $W_{E}$ is supported on $E$, the walk $X_{n}$ is irreducible. Because $W$ is a weighting, relationship (2.1) implies that the natural random walk is reversible with stationary measure $\pi(x)=W_{V}(x) w_{V}(x)$. For the graph arising in Example 2.1, the natural random walk is the usual simple symmetric random on the vertices of the integer lattice in $\mathbb{R}^{n}$.

Associated to $X_{n}$ there is a transition operator $T: C_{0}^{0}(G) \rightarrow C_{0}^{0}(G)$ defined by

$$
T f(x)=\sum_{y \in V} p(x, y) f(y) .
$$

Let $p_{n}(x, y)$ be defined by recursion:

$$
\begin{aligned}
& p_{1}(x, y)=p(x, y), \\
& p_{n}(x, y)=\sum_{z \in V} p(x, z) p_{n-1}(z, y) .
\end{aligned}
$$

Iterating $T$ and using Chapman-Kolmogorov gives

$$
T^{n} f(x)=\sum_{y \in V} p_{n}(x, y) f(y) .
$$

Proposition 2.4. Let $(G, \mathcal{O}, W)$ be a graph with geometry, $\left(X_{n}, P^{x}\right)$ the associated random walk, and $T$ the transition operator associated to $X_{n}$. Let $\Omega_{w}: C_{0}^{0}(G) \rightarrow$ $C_{0}^{0}(G)$ be the multiplication operator defined by $\Omega_{w} f(x)=w_{V}(x) f(x)$. Then

$$
\Delta=\Omega_{w}(I-T),
$$

where $I$ is the identity operator.

Proof. This follows directly from the definition of $p(x, y)$ in $(2.11)$, the definition of $T$ in (2.12), and (2.5).

In the case of manifolds, there is a close relationship between the behavior of a Brownian process in a given domain contained in a Riemannian manifold, and the solutions of boundary value problems for the Laplace operator on the given domain. In the sequel, we present the machinery required to formulate analogs of these notions in the category of graphs with geometry. The material is by no means new and is included for completeness (cf. [C], B], Do, and references therein).

Definition 2.4. Let $(G, \mathcal{O}, W)$ be a graph with natural geometry, $D$ a domain of $G$ with $\partial D \neq \emptyset$. Let $X_{n}$ be the natural random walk associated to $(G, \mathcal{O}, W)$. The index of the first exit time of $X_{n}$ from $i D$ is defined by

$$
\eta=\inf \left\{n: X_{n} \notin i D\right\} .
$$

The first exit time of $X_{n}$ from $i D$ is defined by

$$
\tau=\sum_{n=0}^{\eta-1} \frac{1}{w_{V}\left(X_{n}\right)} .
$$


Note that $\eta$ and $\tau$ are measurable with respect to the filtration associated to $X_{n}$. Our definition of $\tau$ is motivated by our desire to model approximation procedures for the case of manifolds. We view $w_{V}(x)$ as a measure of the "size" of the neighborhood defined by the vertex $x$. Thus our choice reflects our desire to correlate "transition time for leaving a neighborhood defined by a vertex $x$ " with "the size of a neighborhood defined by a vertex $x$."

Note that if for all $x \in i D$ we have $w_{V}(x)=\alpha, \alpha$ a constant, then $\alpha \tau=\eta$, and the walk transitions at a constant rate, proportional to the index.

Definition 2.5. Let $(G, \mathcal{O}, W)$ be a graph with geometry, $D$ a domain of $G$. We say that $D$ is $\alpha$-weight regular if for all $x \in i D$ we have $w_{V}(x)=\alpha$.

Let $(G, \mathcal{O}, W)$ be a graph with geometry, $D$ a domain of $G$ with $\partial D \neq \emptyset$. Because $D$ is finite,

$$
P^{x}\left(X_{n} \in i D \quad \forall n\right)=0 .
$$

In addition, for all natural numbers $k$,

$$
E^{x}\left[\tau^{k}\right]<\infty
$$

Definition 2.6. Let $(G, \mathcal{O}, W)$ be a graph with geometry, $D$ a domain of $G$ with nonempty boundary. Let $X_{n}$ be the process associated to $(G, \mathcal{O}, W)$. Let $x^{*}$ be a cemetery point not contained in $D$. The process $X_{n}$ killed upon leaving $i D$ is the process with transition probabilities $q(x, y)$ given by

$$
q(x, y)= \begin{cases}0 & \text { if } x \notin i D, y \neq x^{*} \\ 1 & \text { if } x \notin i D, y=x^{*} \\ p(x, y) & \text { if } x \in i D .\end{cases}
$$

The transition operator corresponding to the process $X_{n}$ killed upon leaving $i D$, denoted $T_{D}$, is the map $T_{D}: C^{0}(i D) \rightarrow C^{0}(i D)$ defined by

$$
T_{D} f(x)=\sum_{y \in i D} q(x, y) f(y) .
$$

Proposition 2.5. Let $(G, \mathcal{O}, W)$ be a graph with geometry and suppose that $D$ is a domain of $G$ with nonempty boundary. Let $T_{D}$ be the transition operator for the natural random walk killed on exiting $i D$. Let $\langle f, g\rangle_{w}$ be the inner product defined by

$$
\langle f, g\rangle_{w}=\sum_{x \in V} f(x) g(x) W_{V}(x) w_{V}(x) .
$$

Then $T_{D}$ is selfadjoint with respect to the inner product on $C^{0}(i D)$ defined by $(2.19)$, and the eigenvalues of $T_{D}$ all have magnitude less than 1.

Proof. Let $f, g \in C^{0}(i D)$. Then

$$
\begin{aligned}
\left\langle T_{D} f, g\right\rangle_{w} & =\sum_{x \in i D} \sum_{y \in i D} f(y) q(x, y) g(x) W_{V}(x) w_{V}(x) \\
& =\sum_{x \in i D} \sum_{y \in i D} f(y) \frac{W_{E}(x, y)}{w_{V}(x)} g(x) W_{V}(x) w_{V}(x) \\
& =\sum_{x \in i D} \sum_{y \in i D} f(y) \frac{W_{E}(y, x)}{w_{V}(y)} g(x) W_{V}(y) w_{V}(y)=\left\langle f, T_{D} g\right\rangle_{w}
\end{aligned}
$$


proving that $T_{D}$ is selfadjoint. Let $q_{n}$ be defined recursively by

$$
\begin{aligned}
q_{1}(x, y) & =q(x, y), \\
q_{n}(x, y) & =\sum_{z \in V} q(x, z) q_{n-1}(z, y) .
\end{aligned}
$$

Since $P^{x}\left(X_{n} \in i D \forall n\right)=0$, we have that $q_{n}(x, y) \rightarrow 0$ for all $x, y \in D$. From this we conclude that

$$
\lim _{n \rightarrow \infty} T_{D}^{n} f(x)=0
$$

for every $x \in i D$ and every $f \in C^{0}(i D)$. This proves the second claim of the proposition.

Corollary 2.1. Let $(G, \mathcal{O}, W)$ be a graph with geometry and suppose that $D$ is a domain of $G$ with nonempty boundary. Then the interior Laplace operator $\Delta_{i D}$ : $C^{0}(i D) \rightarrow C^{0}(i D)$ is invertible.

Proof. From Proposition 2.3 and Proposition 2.4 we have that

$$
\Delta_{i D}=\Omega_{w}\left(I-T_{D}\right) .
$$

By Proposition 2.5, the eigenvalues of $I-T_{D}$ are contained in the interval $(0,2)$. Since $\Omega_{w}$ is invertible, the corollary follows.

Proposition 2.6. Let $(G, \mathcal{O}, W)$ be a graph with geometry and suppose that $D$ is a domain of $G$ with nonempty boundary. Let $T_{D}$ be the transition operator for the natural random walk killed on exiting $i D$ and let $\eta$ be the first exit index for the natural random walk on $G$. Then, for every positive integer $l$,

$$
P^{x}(\eta=l)=\left[T_{D}^{l-1}\left(I-T_{D}\right)\right]\left(\mathbf{1}_{i D}\right)(x),
$$

where $P^{x}$ is the measure charging paths beginning at $x$ and $\mathbf{1}_{i D}$ is the indicator function of $i D$.

Proof. Note that $\mathbf{1}_{i D}=\sum_{y \in i D} \delta_{y}$ where $\delta_{y}$ is given by (2.6). Hence,

$$
\begin{aligned}
T_{D} \mathbf{1}_{i D}(x) & =\sum_{z \in i D} \sum_{y \in i D} q(x, z) \delta_{y}(z) \\
& =\sum_{y \in i D} q(x, y)
\end{aligned}
$$

where, as above, $q(x, y)$ is the transition probability for the natural random walk killed on leaving $i D$. Since $\sum_{y \in i D} q(x, y)$ is the probability that starting at $x$ we transition to a point in $i D$, we conclude that

$$
\left[I-T_{D}\right]\left(\mathbf{1}_{i D}\right)(x)=P^{x}(\eta=1) .
$$

The proposition now follows from $(2.22)$ and repeated use of the fact that

$$
P^{x}(\eta=2)=\sum_{y \in i D} q(x, y) P^{y}(\eta=1) .
$$


Definition 2.6. Suppose that $(G, \mathcal{O}, W)$ is a graph with geometry and suppose that $D$ is a domain of $G$ with nonempty boundary. Let $T_{D}$ be the transition operator for the natural random walk killed on exiting $i D$. The Green operator of $i D$ is the operator defined by

$$
G=\sum_{n=0}^{\infty} T_{D}^{n}
$$

Note that by Proposition 2.5 the sum in (2.23) converges and $G$ is well defined. Indeed, we note that the Green operator gives the inverse of the Laplace operator $\Delta_{i D}$ : For every $f \in C^{0}(i D)$ and every $x \in i D$,

$$
\Delta_{i D} G \Omega_{w}^{-1} f(x)=f(x) .
$$

\section{Discrete Boundary Value Problems}

In this section, we develop the machinery required to prove Theorem 1.1. Throughout this section, $(G, \mathcal{O}, W)$ will be a graph with geometry (cf. Definition 2.1 ) and $D$ will be a domain of $G$ with nonempty boundary (cf. Definition 2.2).

Let $\Delta_{i D}$ be the interior Laplace operator associated to $D$ (cf. (2.10)). Define a sequence of functions $f_{k} \in C^{0}(i D)$ recursively as follows: Set

$$
f_{0}(x)=1 \text { for all } x \in i D
$$

and, having defined $f_{j}, 1 \leq j<k$, let $f_{k}: i D \rightarrow \mathbb{R}$ be the unique solution of

$$
\Delta_{i D} f_{k}+k f_{k-1}+\sum_{j=2}^{k}\left(\begin{array}{l}
k \\
j
\end{array}\right)\left(-\frac{1}{w_{V}}\right)^{j-1} f_{k-j}=0,
$$

where $w_{V}$ is the natural auxiliary weighting for the graph with geometry $(G, \mathcal{O}, W)$ (cf. (2.2)).

The functions $f_{k}$ are closely related to the exit time moments (from $D$ ) of the natural random walk associated to $(G, \mathcal{O}, W)$ :

Theorem 3.1. Let $D$ be a domain and, for $k$ a nonnegative integer, let $f_{k}$ be defined by (3.1) and (3.2) above. Let $X_{n}$ be the natural random walk on the underlying graph and suppose that $P^{x}$ is the associated measure charging paths beginning at $x$. Let $\tau$ be the expected exit time (from $D$ ) of the natural random walk as defined by (2.16). Then

$$
f_{k}(x)=E^{x}\left[\tau^{k}\right]
$$

where $E^{x}$ is the expectation corresponding to the measure $P^{x}$.

Proof. From Proposition 2.3 and the definition of the transition probabilities we have

$$
\begin{aligned}
-\Delta_{i D} E^{x}\left[\tau^{k}\right] & =\left[\sum_{y \in i D} W_{E}(x, y) E^{y}\left[\tau^{k}\right]-w_{V}(x) E^{x}\left[\tau^{k}\right]\right] \\
& =w_{V}(x)\left[\sum_{y \in i D} p(x, y) E^{y}\left[\tau^{k}\right]-E^{x}\left[\tau^{k}\right]\right] .
\end{aligned}
$$


But

$$
E^{y}\left[\tau^{k}\right]=\sum_{l=0}^{\infty}\left(\sum_{n=0}^{l-1} \frac{1}{w_{V}\left(X_{n}\right)}\right)^{k} P^{y}(\eta=l)
$$

where $X_{n}$ is the natural random walk, $P^{y}$ is the measure charging paths beginning at $y$, and $\eta$ is as defined in (2.15). Hence,

$$
\sum_{y \in i D} p(x, y) E^{y}\left[\tau^{k}\right]=\sum_{l=0}^{\infty} \sum_{y \in i D}\left(\sum_{n=0}^{l-1} \frac{1}{w_{V}\left(X_{n}^{y}\right)}\right)^{k} p(x, y) P^{y}(\eta=l),
$$

where the " $y$ " superscript emphasizes that the paths contributing to the sum begin at $y$. Noting that, for $l \geq 1$,

$$
P^{x}(\eta=l+1)=\sum_{y \in i D} p(x, y) P^{y}(\eta=l)
$$

we have

$$
\sum_{y \in i D} p(x, y) E^{y}\left[\tau^{k}\right]=\sum_{l=1}^{\infty}\left(\left(\sum_{n=0}^{l} \frac{1}{w_{V}\left(X_{n}^{x}\right)}\right)-\frac{1}{w_{V}(x)}\right)^{k} P^{x}(\eta=l+1) .
$$

From (3.4) and (3.5) we conclude that

$$
\Delta_{i D} E^{x}\left[\tau^{k}\right]=w_{V}(x)\left[E^{x}\left[\left(\tau-\left(w_{V}(x)\right)^{-1}\right)^{k}\right]-E^{x}\left[\tau^{k}\right]\right] .
$$

Theorem 3.1 follows immediately from (3.6).

The relationship between the solutions of the Poisson problems defined by (3.1)(3.2) and the exit time moments for the natural random walk on the underlying graph are closely related to the corresponding relationship between the behavior of Brownian motion in a domain in a Riemannian manifold $M$ and the solutions of a hierarchy of Poisson problems on the domain. More precisely, suppose that $D \subset M$ is a smoothly bounded open domain with compact closure. Let $\tau$ be the first exit time of classical Brownian motion from $D$. Suppose that $\Delta$ is the Laplace operator on $M$ and define a sequence of functions $u_{k}$ recursively as follows: Let

$$
\begin{aligned}
& u_{0}(x)=1 \text { for all } x \in D, \\
& u_{0}(x)=0 \text { for all } x \in \partial D,
\end{aligned}
$$

and, having defined $u_{j}$ for $0 \leq j<k$, let $u_{k}$ be the solution to the Poisson problem

$$
\begin{aligned}
\frac{1}{2} \Delta u_{k}+k u_{k-1} & =0 \text { for all } x \in D, \\
u_{k}(x) & =0 \text { for all } x \in \partial D .
\end{aligned}
$$

Then (cf. $[\underline{\mathrm{H}}], \underline{\mathrm{M}}]$ )

$$
u_{k}(x)=E^{x}\left[\tau^{k}\right]
$$

where $E^{x}$ is expectation with respect to the measure weighting paths beginning at $x$.

Given (3.9), the sum appearing in (3.2) can be interpreted as the correction necessary to insure that exit time moments for the natural walk can be expressed as solutions to discrete Poisson problems on the corresponding domain. Note that this correction term vanishes for the first moment. 
As mentioned in the introduction (cf. (1.9) and (1.10)), the quantities

$$
\begin{aligned}
B_{1, k}(D) & =\int_{D} E^{x}\left[\tau^{k}\right] d x \\
& =\int_{D} u_{k}(x) d x
\end{aligned}
$$

are invariants of the underlying metric which can be computed via variational principles. In the case $k=1$ the Poisson problems which compute the expected exit time for both the discrete case (cf. (3.2)) and the smooth case (cf. (3.8)) have the same form, and we expect to be able to formulate and prove the discrete analog of (1.11). When $k \neq 1$, the structures of the corresponding Poisson problems differ, and we expect combinatorial correction terms to arise. We proceed to determine the precise form of the correction.

Let $D$ be a domain in a graph with geometry and define a sequence of functions recursively as follows: Let

$$
g_{0}=1 \text { for all } x \in i D
$$

and, having defined $g_{j}$ for $0 \leq j<k$, let $g_{k}$ be the unique solution to the Poisson problem

$$
\Delta_{i D} g_{k}+k g_{k-1}=0 \text { for all } x \in i D .
$$

Theorem 3.2. Suppose that $D$ is a domain with nonempty boundary and that $\Delta_{i D}$ is the interior Laplace operator associated to D. For $k$ a nonnegative integer, let $g_{k}$ be defined by (3.10)-(3.11). Then, with the pairings defined by (2.3),

$$
\left\langle g_{k}, 1\right\rangle_{i D}=k ! \sup _{g \in C^{0}(i D) \backslash\{0\}} \frac{\langle g, 1\rangle_{i D}^{2}}{\left|\left\langle g, \Delta_{i D}^{k} g\right\rangle_{i D}\right|} .
$$

Proof. The proof follows the argument given in [KM]. For $g \in C^{0}(i D) \backslash\{0\}$, consider the quotient

$$
Q_{k}(g)=\frac{\langle 1, g\rangle_{i D}^{2}}{\left|\left\langle g, \Delta_{i D}^{k} g\right\rangle_{i D}\right|} .
$$

Note that $Q_{k}$ is invariant under scaling: for all nonzero scalars $z$,

$$
Q_{k}(z g)=Q_{k}(g) .
$$

Suppose $k=2 m$. Since $\Delta_{i D}$ is selfadjoint, we have

$$
Q_{k}(g)=\frac{\langle 1, g\rangle_{i D}^{2}}{\left\|\Delta_{i D}^{m} g\right\|_{i D}^{2}} .
$$

Since $\Delta_{i D}$ is invertible we can write $g=\Delta_{i D}^{-m} h$ for some $h \in C^{0}(i D)$. Since $\Delta_{i D}$ is selfadjoint and $Q_{k}$ is scale invariant (3.12), we conclude that

$$
\sup _{g \in C^{0}(i D) \backslash\{0\}} Q_{k}(g)=\sup _{h \in C^{0}(i D),\|h\|_{i D}^{2}=1}\left\langle\Delta_{i D}^{-m}(1), h\right\rangle_{i D}^{2} .
$$

To maximize the inner product appearing on the right-hand side of (3.13), we take the unit vector in the direction of $\Delta_{i D}^{-m}(1)$. That is, we set $h=\frac{\Delta_{i D}^{-m}(1)}{\left\|\Delta_{i D}^{-m}(1)\right\|}$, 
$g=\frac{\Delta_{i D}^{-2 m}(1)}{\left\|\Delta_{i D}^{-m}(1)\right\|}$, and use scale invariance to write

$$
\begin{aligned}
\sup _{g \in C^{0}(i D) \backslash\{0\}} Q_{k}(g) & =Q_{k}\left(\frac{\Delta_{i D}^{-2 m}(1)}{\left\|\Delta_{i D}^{-m}(1)\right\|_{i D}}\right) \\
& =\frac{\left\langle 1, \Delta_{i D}^{-2 m}(1)\right\rangle_{i D}^{2}}{\left|\left\langle\Delta_{i D}^{-2 m}(1), \Delta_{i D}^{2 m} \Delta_{i D}^{-2 m}(1)\right\rangle_{i D}\right|} .
\end{aligned}
$$

From the recursion (3.10)-(3.11) defining the $g_{k}$ we have

$$
\Delta_{i D}^{-k}(1)=\frac{(-1)^{k}}{k !} g_{k} \text {. }
$$

Using (3.15), we obtain

$$
\sup _{g \in C^{0}(i D) \backslash\{0\}} Q_{k}(g)=\frac{1}{(2 m) !}\left\langle 1, g_{2 m}\right\rangle_{i D},
$$

which concludes the proof of the theorem when $k$ is even. The argument for odd $k$ is similar: Suppose that $k=2 m+1$, and write

$$
Q_{k}(g)=\frac{\langle 1, g\rangle_{i D}^{2}}{\left|\left\langle\Delta_{i D}^{m} g, \Delta_{i D} \Delta_{i D}^{m} g\right\rangle_{i D}\right|} .
$$

Since $\Delta_{i D}=d_{W}^{*} d$, we have

$$
Q_{k}(g)=\frac{\langle 1, g\rangle_{i D}^{2}}{\left|\left\langle d \Delta_{i D}^{m} g, d \Delta_{i D}^{m} g\right\rangle_{i D}\right|},
$$

where the pairing in the denominator is understood to be on edge functions. As above, set $g=\Delta_{i D}^{-m} h$ and note that

$$
\sup _{g \in C^{0}(i D) \backslash\{0\}} Q_{k}(g)=\sup _{h \in C^{0}(i D),\|d h\|_{i D}^{2}=1}\left\langle d \Delta_{i D}^{-m-1}(1), d h\right\rangle_{i D}^{2},
$$

where once again the pairing is for edge functions. As before, to maximize the inner product appearing on the right-hand side of (3.17), we take the unit vector in the direction of $d \Delta_{i D}^{-m-1}(1)$. That is, we set $d h=\frac{d \Delta_{i D}^{-m-1}(1)}{\left\|d \Delta_{i D}^{-m-1}(1)\right\|}$ and use scale invariance to write

$$
\sup _{g \in C^{0}(i D) \backslash\{0\}} Q_{k}(g)=\frac{\left\langle d \Delta_{i D}^{-m-1}(1), d \Delta_{i D}^{-m-1}(1)\right\rangle_{i D}^{2}}{\left|\left\langle d \Delta_{i D}^{-m-1}(1), d \Delta_{i D}^{-m-1}(1)\right\rangle_{i D}\right|} .
$$

Integrating by parts and using (3.18), we obtain

$$
\sup _{g \in C^{0}(i D) \backslash\{0\}} Q_{k}(g)=\frac{1}{(2 m+1) !}\left\langle 1, g_{2 m+1}\right\rangle_{i D},
$$

which concludes the proof of the theorem when $k$ is odd.

\section{Proof of Theorem 1.1}

Throughout this section $(G, \mathcal{O}, W)$ will be a graph with natural geometry and $D \subset G$ will be a domain with nonempty boundary which is $\alpha$-weight regular (cf. Definition (2.5)).

As above, let $X_{n}$ be the natural random walk on $G$, let $\eta$ be the first exit index of $X_{n}$ from $D$ (cf. (2.15)), and $\tau$ the first exit time of $X_{n}$ from $D$ (cf. (2.16)). As a consequence of $\alpha$-weight regularity, we have

$$
\eta=\alpha \tau \text {. }
$$


To prove Theorem 1.1 we will express the functions $E^{x}\left[\tau^{k}\right]$ in terms of the functions $g_{k}(x)$ defined by (3.10)-(3.11). With this in mind, consider the power series

$$
P_{k}(x)=x \sum_{l=1}^{\infty} l^{k}(1-x)^{l-1},
$$

which converges absolutely for $x \in(0,2)$. There is a simple recursion for $P_{k}(x)$ :

$$
\begin{aligned}
P_{0}(x) & =1, \\
P_{k+1}(x) & =\frac{1}{x} P_{k}(x)+(x-1) \frac{d}{d x} P_{k}(x) .
\end{aligned}
$$

The following definition will provide convenient notation:

Definition 4.1. Let $n$ and $k$ be positive integers. The Stirling numbers of the second kind are defined by

$$
\left\{\begin{array}{l}
k \\
n
\end{array}\right\}=\frac{(-1)^{n}}{n !} \sum_{m=1}^{n}\left(\begin{array}{c}
n \\
m
\end{array}\right)(-1)^{m} m^{k} .
$$

The Stirling numbers of the first kind are defined by

$$
\left[\begin{array}{l}
k \\
n
\end{array}\right]=\sum_{m=0}^{k-n}(-1)^{m}\left(\begin{array}{c}
k-1+m \\
k-n+m
\end{array}\right)\left(\begin{array}{c}
2 k-n \\
k-n-m
\end{array}\right)\left\{\begin{array}{c}
k-n-m \\
m
\end{array}\right\} .
$$

Recall that the Stirling numbers of the first kind count the number of permutations of $k$ symbols which contain exactly $n$ symbols, while the Stirling numbers of the second kind represent the number of ways of partitioning $k$ objects into $n$ blocks. These numbers play a role in combinatorics and statistical mechanics (cf. [Z]).

Lemma 4.1. For $k \geq 1$,

$$
P_{k}(x)=(-1)^{k} \sum_{n=1}^{k}\left\{\begin{array}{l}
k \\
n
\end{array}\right\} n !\left(-\frac{1}{x}\right)^{n},
$$

where $\left\{\begin{array}{l}k \\ n\end{array}\right\}$ is the Stirling number of the second kind.

Proof. We check the case $k=1$ by starting with (4.1), summing the geometric series, and differentiating. The general case follows by induction using the recursion (4.2).

Lemma 4.2. Let $D$ be an $\alpha$-weight regular domain. Then, with notation as above,

$$
E^{x}\left[\tau^{k}\right]=\sum_{n=1}^{k}\left\{\begin{array}{l}
k \\
n
\end{array}\right\}(-\alpha)^{n-k} n ! \Delta_{i D}^{-n}\left(\mathbf{1}_{i D}\right)(x) .
$$

Proof. From the definition we have

$$
\begin{aligned}
E^{x}\left[\tau^{k}\right] & =\sum_{l=1}^{\infty}\left(\sum_{n=0}^{l-1} \frac{1}{w_{V}\left(X_{n}\right)}\right)^{k} P^{x}(\eta=l) \\
& =\alpha^{-k} \sum_{l=1}^{\infty} l^{k} P^{x}(\eta=l) .
\end{aligned}
$$


Using Proposition 2.6 (cf. (2.21)), we obtain

$$
E^{x}\left[\tau^{k}\right]=\alpha^{-k} \sum_{l=1}^{\infty} l^{k}\left[T_{D}^{l-1}\left(I-T_{D}\right)\right]\left(\mathbf{1}_{i D}\right)(x) .
$$

By (2.20) and $\alpha$-weight regularity,

$$
T_{D}=I-\alpha^{-1} \Delta_{i D}
$$

Using (4.7), (4.8), and (4.1), we obtain

$$
\begin{aligned}
E^{x}\left[\tau^{k}\right] & =\alpha^{-k} \sum_{l=1}^{\infty} l^{k}\left[\left(I-\alpha^{-1} \Delta_{i D}\right)^{l-1} \alpha^{-1} \Delta_{i D}\right]\left(\mathbf{1}_{i D}\right)(x) \\
& =\alpha^{-k} P_{k}\left(\alpha^{-1} \Delta_{i D}\right)\left(\mathbf{1}_{i D}\right)(x) .
\end{aligned}
$$

Using (4.9) and Lemma 4.1, we obtain

$$
E^{x}\left[\tau^{k}\right]=\alpha^{-k}(-1)^{k} \sum_{n=1}^{k}\left\{\begin{array}{l}
k \\
n
\end{array}\right\} n !\left(-\frac{1}{\alpha^{-1} \Delta_{i D}}\right)^{n}\left(\mathbf{1}_{i D}\right)(x),
$$

from which the lemma follows.

Theorem 4.1. Let $D$ be an $\alpha$-weight regular domain with nonempty boundary. Let $\tau$ be the first exit time of the natural random walk from the domain $D$ and let $g_{k}$ be the solutions of the Poisson problems defined recursively by (3.10)-(3.11). Then

$$
E^{x}\left[\tau^{k}\right]=g_{k}+\sum_{n=1}^{k-1}\left\{\begin{array}{l}
k \\
n
\end{array}\right\}(-\alpha)^{n-k} g_{n}
$$

where $\left\{\begin{array}{l}k \\ n\end{array}\right\}$ are the Stirling numbers of the second kind (4.3)

Proof. The recursion (3.10)-(3.11) defining $g_{k}$ gives

$$
g_{n}(x)=(-1)^{n} n ! \Delta_{i D}^{-n}\left(\mathbf{1}_{i D}\right)(x) .
$$

Noting that $\left\{\begin{array}{l}k \\ k\end{array}\right\}=1$ and using Lemma 4.2 , we complete the proof of the theorem.

Proof of Theorem 1.1. Pairing both sides of (4.11) gives (1.7). To see that (1.8) holds, note that the Stirling numbers satisfy the following: If we define a polynomial $x^{(k)}$ by

$$
x^{(k)}=x(x-1)(x-2) \cdot(x-k+1)
$$

then (cf. $[\mathrm{Z}]$ ),

$$
x^{(k)}=\sum_{n=1}^{k}\left[\begin{array}{l}
k \\
n
\end{array}\right] x^{n}
$$

and

$$
x^{k}=\sum_{n=1}^{k}\left\{\begin{array}{l}
k \\
n
\end{array}\right\} x^{(n)} .
$$

Thus, (1.8) follows from (1.7), (4.13) and (4.14), which completes the proof of Theorem 1.1. 


\section{Spectra and Graph Geometry}

In this section we give proofs for Theorem 1.2 and Theorem 1.3. We begin with a definition:

Definition 5.1. Let $(G, \mathcal{O}, W)$ be a graph with geometry and suppose that $D$ is a domain in $G$ with nonempty boundary. Suppose that $\operatorname{spec}(D)$ is the spectrum of the interior Laplace operator on $D$ and suppose that $\lambda_{j} \in \operatorname{spec}(D)$. Let $E_{\lambda_{j}}$ be the eigenspace corresponding to $\lambda_{j}$ and let $\left\{\phi_{\lambda_{j}, l}: 1 \leq l \leq \operatorname{dim}\left(E_{\lambda_{j}}\right)\right\}$ be an orthonormal basis of $E_{\lambda_{j}}$. Let

$$
\hat{a}_{j}^{2}=\sum_{l=1}^{\operatorname{dim}\left(E_{\lambda_{j}}\right)}\left\langle\phi_{j, l}, \mathbf{1}_{i D}\right\rangle
$$

be the projection of $E_{\lambda_{j}}$ on the space of constant functions. We call the sequence

$$
a^{2}=\left(\hat{a}_{1}^{2}, \hat{a}_{2}^{2}, \ldots, \hat{a}_{j}^{2}, \ldots\right)
$$

a spectral partition of the volume of $D$.

We note that a spectral partition of volume is independent of the choice of basis for each eigenspace.

The following will be used on a number of occasions:

Lemma 5.1. Suppose that $(G, \mathcal{O}, W)$ is a graph with geometry and that $D$ is a domain of $G$ with nonempty boundary. Let $g_{k}$ be defined recursively as solutions to Poisson problems by (3.10)-(3.11). Suppose that $\operatorname{spec}(D)$ is the spectrum of the interior Laplace operator associated to $D$ and that $\phi_{j}$ is a normalized eigenfunction corresponding to the eigenvalue $\lambda_{j}$ (where we take an orthonormal basis of eigenfunctions when the multiplicity is greater than one). Let

$$
a_{j}=\left\langle\mathbf{1}_{i D}, \phi_{j}\right\rangle_{V}
$$

where $\mathbf{1}_{i D}$ is the indicator function of $i D$. Then

$$
\left\langle g_{k}, \phi_{j}\right\rangle_{V}=\frac{(-1)^{k} k !}{\lambda_{j}^{k}} a_{j} .
$$

Proof. With notation as above, we have

$$
\mathbf{1}_{i D}=\sum_{j} a_{j} \phi_{j}
$$

Using that $g_{k}$ is defined by the recursion (3.10)-(3.11) and that $\Delta_{i D}$ is selfadjoint, we obtain

$$
\begin{aligned}
\left\langle g_{k}, \phi_{j}\right\rangle_{V} & =\lambda_{j}^{-1}\left\langle g_{k}, L \phi_{j}\right\rangle_{V} \\
& =\frac{-k}{\lambda_{j}}\left\langle g_{k-1}, \phi_{j}\right\rangle_{V} .
\end{aligned}
$$

Continuing inductively and using (5.4), we obtain (5.3).

Corollary 5.1. Let $(G, \mathcal{O}, W)$ be a graph with geometry and suppose that $D$ is a domain in $G$ with nonempty boundary. Let $\operatorname{spec}(D)$ be the spectrum of the interior Laplace operator on $D$ and suppose that $\operatorname{spec}^{*}(D)$ is given by $(1.12)$. Let $\operatorname{mspec}(D)$ and $\operatorname{pspec}(D)$ be given by (1.5) and (1.6), respectively. Then $\operatorname{pspec}(D)$ is determined by $\operatorname{spec}^{*}(D)$ and the spectral partition of the volume. When $D$ is $\alpha$-weight regular, the same claims hold with $\operatorname{pspec}(D)$ replaced by $\operatorname{mspec}(D)$. 
Proof. For each distinct eigenvalue, fix an orthonormal basis of the corresponding eigenspace. Let $a^{2}=\left(\hat{a}_{1}^{2}, \hat{a}_{2}^{2}, \ldots, \hat{a}_{j}^{2}, \ldots\right)$ be a spectral partition of volume as defined in (5.1). From Lemma 5.1 and Theorem 3.2, we have

$$
A_{2, k}=\sum_{j}\left(-\frac{1}{\lambda_{j}}\right)^{k} k ! \hat{a}_{j}^{2},
$$

where the sum is over elements of $\operatorname{spec}^{*}(D)$. The claims of the corollary concerning elements of $\operatorname{pspec}(D)$ follow from (5.5). By Theorem 1.1, the same is true for $\operatorname{mspec}(D)$ when $D$ is $\alpha$-weight regular.

In what follows, we construct a partial converse for Corollary 5.1.

Proof of Theorem 1.2. Let $\left\{\mu_{j}\right\}$ be positive real numbers. The Problem of Moments as formulated by Stieltjes asks: For which sequences $\left\{\mu_{j}\right\}$ is it possible to find a bounded nondecreasing function $\psi:[0, \infty) \rightarrow \mathbb{R}$ such that

$$
\mu_{j}=\int_{0}^{\infty} x^{j} d \psi(x) ?
$$

The problem arises in a variety of contexts (probability and statistics, orthogonal polynomials, mechanics, etc.) and has an extensive associated literature which begins with a series of papers by Tchebycheff starting in 1855 (cf. $[\mathrm{SH}$ for background on the moment problem). In 1894-95, Stieltjes gave a treatment of the problem (in which he developed the notion of the Stieltjes integral) and clarified under what conditions the problem of moments admits a solution. We recall the relevant ideas and notation.

For $n$ a nonnegative integer, let $M_{0, n}$ be the $n \times n$ symmetric square matrix defined by

$$
\left(M_{0, n}\right)_{i j}=\mu_{i+j}, \quad 0 \leq i, j \leq n-1 .
$$

Let

$$
\left(M_{1, n}\right)_{i j}=\mu_{i+j+1}, \quad 0 \leq i, j \leq n-1 .
$$

We have the following:

Theorem (cf. $[\mathrm{SH}])$. Let $\left\{\mu_{j}\right\}$ be positive real numbers. A necessary condition for the existence of a solution of the Stieltjes moment problem

$$
\mu_{j}=\int_{0}^{\infty} x^{j} d \psi(x)
$$

is that

$$
\operatorname{det}\left(M_{0, n}\right) \geq 0, \quad \operatorname{det}\left(M_{1, n}\right) \geq 0 \text { for all } n .
$$

In order that the spectrum of the problem not reduce to a finite set it is necessary and sufficient that

$$
\operatorname{det}\left(M_{0, n}\right)>0, \quad \operatorname{det}\left(M_{1, n}\right)>0 \text { for all } n .
$$

In order that there exists a solution whose spectrum is exactly $k+1$ points distinct from 0 , it is necessary and sufficient that

$$
\begin{array}{ll}
\operatorname{det}\left(M_{0, n}\right)>0, & \operatorname{det}\left(M_{1, n}\right)>0 \text { for all } n, 0 \leq n \leq k, \\
\operatorname{det}\left(M_{0, n}\right)=0, & \operatorname{det}\left(M_{1, n}\right)=0 \text { for all } n>k .
\end{array}
$$

In the last case, $\psi$ is uniquely determined by the sequence $\left\{\mu_{j}\right\}$. 
For $N$ fixed, let $\mathcal{D}_{N}$ be as defined in (1.13). Fix $D \in \mathcal{D}_{N}$ and choose

$$
\mu_{n}=\frac{A_{2, n}}{n !} .
$$

Let $\hat{a}_{j}^{2}$ be the elements of a spectral partition of volume as defined in (5.1). Then, from (5.5), we have

$$
\mu_{n}=\sum_{j=1}^{N}\left(-\frac{1}{\lambda_{j}}\right)^{n} \hat{a}_{j}^{2},
$$

where the sum runs over $j$ for which $\lambda_{j} \in \operatorname{spec}^{*}(D)$. Define $\psi(x)$ by

$$
\psi(x)=\sum_{j=1}^{N} \hat{a}_{j}^{2} \mathbf{1}_{\left[-\frac{1}{\lambda_{j}}, \infty\right)}(x) .
$$

Clearly, $\psi(x)$ is the unique solution to the moment problem, and hence the conditions given in (5.9) hold, where $N$ is the number of elements in the set $\operatorname{spec}^{*}(D)$. Since $\operatorname{pspec}(D)$ determines the set $\left\{\mu_{j}\right\}$, this implies that the Poisson spectrum determines $\operatorname{spec}^{*}(D)$, as claimed in Theorem 1.2. By Theorem 1.1, when $D$ is $\alpha$-weight regular, the same is true for $\operatorname{mspec}(D)$.

To understand the precise relationship between the Poisson spectrum and $\operatorname{spec}^{*}(D)$, let $X$ be the vector $\left(X_{1}, X_{2}, \ldots, X_{N}\right)$ and consider the Vandermonde matrix

$$
V_{X}=\left(\begin{array}{cccc}
1 & 1 & \ldots & 1 \\
X_{1} & X_{2} & \ldots & X_{N} \\
\vdots & \vdots & \vdots & \vdots \\
X_{1}^{N-1} & X_{2}^{N-1} & \ldots & X_{N}^{N-1}
\end{array}\right) .
$$

Note that when the $X_{i}$ are distinct, $V_{X}$ is invertible. Let $\mu_{n}$ be defined as in (5.10) and set

$$
\mu=\left(\mu_{0}, \mu_{1}, \ldots, \mu_{N-1}\right) .
$$

Let $\left\{\lambda_{j}\right\}=\operatorname{spec}^{*}(D)$ and write

$$
-\lambda^{-1}=\left(-\frac{1}{\lambda_{1}},-\frac{1}{\lambda_{2}}, \ldots,-\frac{1}{\lambda_{N}}\right) .
$$

Let $a^{2}$ be the spectral partition of volume defined as in (5.1):

$$
a^{2}=\left(\hat{a}_{1}^{2}, \hat{a}_{2}^{2}, \ldots \hat{a}_{N}^{2}\right) .
$$

By (5.10) we have

$$
\mu=V_{-\lambda^{-1}} a^{2} .
$$

Let $-\Lambda^{-1}$ be the diagonal matrix defined by $-\lambda^{-1}$. Let

$$
b_{j}=\left(\mu_{j}, u_{j+1}, \ldots, u_{j+N-1}\right)^{\mathrm{T}},
$$

where the superscript denotes transpose. Then $b_{n}=V_{-\lambda^{-1}}\left(-\Lambda^{-1}\right)^{n} a^{2}$ and, since the $\lambda_{j}$ are distinct (this is the point of introducing the spectral partition of volume), $V_{-\lambda^{-1}}$ is invertible. Thus, we have

$$
b_{j}=V_{-\lambda^{-1}}\left(-\Lambda^{-1}\right) V_{-\lambda^{-1}}^{-1} b_{j-1} .
$$


Thinking of the vectors $b_{n}$ as columns of a matrix and using (5.17) repeatedly, we obtain

$$
M_{1, N}=V_{-\lambda^{-1}}\left(-\Lambda^{-1}\right) V_{-\lambda^{-1}}^{-1} M_{0, N},
$$

where $M_{0, N}$ and $M_{1, N}$ are given by (5.7) and (5.8), respectively. Since $M_{0, N}$ is invertible, we obtain

$$
V_{-\lambda^{-1}}\left(-\Lambda^{-1}\right) V_{-\lambda^{-1}}^{-1}=M_{1, N}\left(M_{0, N}\right)^{-1}
$$

The characteristic polynomial of $-\Lambda^{-1}$ is invariant under conjugation by an invertible matrix. Thus, the polynomial

$$
\begin{aligned}
P_{D}(x) & =\prod_{j}\left(-\frac{1}{\lambda_{j}}-x\right) \\
& =\operatorname{det}\left(M_{1, N}\left(M_{0, N}\right)^{-1}-x I\right)
\end{aligned}
$$

has coefficients which are polynomial in the entries of the matrix $M_{1, N}\left(M_{0, N}\right)^{-1}$. In particular, we can conclude that $\operatorname{spec}^{*}(D)$ is determined by the roots of a polynomial whose coefficients are rational functions of the $\mu_{j}$. We conclude that the polynomial appearing in (5.20) satisfies the claims of Theorem 1.2, which concludes the proof of the theorem.

Corollary 5.2. Let $(G, \mathcal{O}, W)$ be a graph with geometry and suppose that $D$ is a domain in $G$ with nonempty boundary. Let $a^{2}$ be the spectral partition of volume for $D$ as defined in (5.1). Then $\operatorname{pspec}(D)$ determines $a^{2}$.

Proof. By Theorem 1.2, $\operatorname{pspec}(D)$ determines $\operatorname{spec}^{*}(D)$. Hence, $\operatorname{pspec}(D)$ determines the Vandermonde matrix given by (5.12), as well as its inverse. The corollary now follows from (5.16).

Definition 5.2. Suppose that $(G, \mathcal{O}, W)$ is a graph with geometry and that $D$ is a domain of $G$. Suppose that $H(x, t)$ is a solution to the following boundary value problem with initial data:

$$
\begin{aligned}
\Delta_{i D} H & =\partial_{t} H \text { on } i D \times(0, \infty), \\
H(x, 0) & =1 \text { on } i D \\
H(y, t) & =0 \text { on } \partial D \times(0, \infty) .
\end{aligned}
$$

The heat content of $D$ is the function $Q(t)$ defined by

$$
Q(t)=\left\langle H, \mathbf{1}_{i D}\right\rangle_{V},
$$

where $\mathbf{1}_{i D}$ is the indicator function of $i D$.

The heat content admits a power series expansion:

$$
Q(t) \simeq \sum_{n=0}^{\infty} q_{n} t^{n}
$$

The coefficients $q_{n}$ in the expansion (5.23) are called the heat content asymptotics for the domain $D$. In the remainder of this paper, we investigate the relationship between heat content asymptotics and spectra. 
Proof of Theorem 1.3. Let $(G, \mathcal{O}, W)$ be a graph with geometry and suppose that $D$ is a domain of $G$ with nonempty boundary. Suppose that $H(x, t)$ solves (5.21). Then, with the eigenvalues of $\Delta_{i D}$ denoted by $\lambda_{j}$, the corresponding normalized eigenfunctions denoted by $\phi_{j}$, and $a_{j}$ defined by (5.2), we have

$$
H(x, t)=\sum_{j} a_{j} \phi_{j}(x) e^{\lambda_{j} t},
$$

where the sum is over $j$ with $\lambda_{j} \in \operatorname{spec}(D)$. Thus,

$$
Q(t)=\sum_{j} \hat{a}_{j}^{2} e^{\lambda_{j} t}
$$

where the $\hat{a}_{j}^{2}$ are elements of a spectral partition of volume and the sum is over $\lambda_{j} \in \operatorname{spec}^{*}(D)$. Using (5.25) and power series expansions of $e^{\lambda_{j} t}$, we see that the heat content asymptotics are given by

$$
q_{n}=\sum_{j} \hat{a}_{j}^{2} \frac{\lambda_{j}^{n}}{n !}
$$

where once again the sum is over $j$ with $\lambda_{j} \in \operatorname{spec}^{*}(D)$. The second claim of Theorem 1.3 now follows from (5.26), Theorem 1.2, and Corollary 5.2.

Given (5.3), and the similarity to (5.10), it is instructive to follow the technique introduced in the proof of Theorem 1.2 to study the relationship of the heat content asymptotics to $\operatorname{spec}^{*}(D)$. To this end, let

$$
\mu_{n}=(-1)^{n} n ! q_{n} .
$$

Then $\mu_{n}$ is positive, and we have

$$
\mu_{n}=\sum_{j}\left(-\lambda_{j}\right)^{n} \hat{a}_{j}^{2}
$$

Let $-\lambda=\left(-\lambda_{1},-\lambda_{2}, \cdots-\lambda_{N}\right)$, let $a^{2}$ be defined as in (5.1) and let $\mu$ be defined as in (5.13). Then we have

$$
\mu=V_{-\lambda} a^{2}
$$

where $V_{-\lambda}$ is the Vandermonde matrix given by (5.12). Let $\Lambda_{-\lambda}$ be the diagonal matrix defined by $-\lambda$. Setting $c_{j}=\left(\mu_{j}, \mu_{j+1}, \ldots, \mu_{j+N}\right)^{T}$, assuming that $V_{-\lambda}$ is invertible, and following our previous computation, we have

$$
c_{j}=V_{-\lambda} \Lambda_{-\lambda}\left(V_{-\lambda}\right)^{-1} c_{j-1},
$$

which leads immediately to

$$
M_{1, N}=V_{\lambda} \Lambda_{\lambda} V_{\lambda}^{-1} M_{0, N}
$$

where $M_{0, N}$ and $M_{1, N}$ are given by (5.6) and (5.7), respectively. As before, we can construct an explicit solution to the corresponding Stieltjes Moment Problem:

$$
\psi(x)=\sum_{j} \hat{a}_{j}^{2} \mathbf{1}_{\left[-\lambda_{j}, \infty\right)}(x) .
$$

As before, when $M_{0, N}$ is invertible, we obtain

$$
V_{\lambda} \Lambda_{\lambda} V_{\lambda}^{-1}=M_{1, N}\left(M_{0, N}\right)^{-1} .
$$

From (5.28) we conclude that the heat content asymptotics determine $\operatorname{spec}^{*}(D)$, proving the first claim of Theorem 1.3. Our computations allow us to conclude that, as in the statement of Theorem 1.2, for $N$ fixed, there are $N$ rational functions 
$f_{i}^{\prime}, 1 \leq i \leq N, f_{i}^{\prime}: \mathbb{R}^{2 N} \rightarrow \mathbb{R}$, such that for every $D \in \mathcal{D}_{N}$, the roots of the polynomial

$$
P_{N}^{\prime}(x)=x^{N}+\sum_{i=0}^{N-1} f_{i}^{\prime}\left(A_{2,0}(D), A_{2,1}(D), \ldots, A_{2,2 N-1}(D)\right) x^{i}
$$

give the elements of $\operatorname{spec}^{*}(D)$, which concludes the proof of Theorem 1.3.

We note that the heat content asymptotics are determined by $\operatorname{spec}^{*}(D)$ and the spectral partition of volume:

Corollary 5.3. Let $(G, \mathcal{O}, W)$ be a graph with geometry and suppose that $D$ is a domain in $G$ with nonempty boundary. Let $\operatorname{spec}(D)$ be the spectrum of the interior Laplace operator on $D$ and suppose that $\operatorname{spec}^{*}(D)$ is given by (1.12). Let $\left\{q_{n}\right\}$ be the heat content asymptotics of $D$. Then $\left\{q_{n}\right\}$ is determined by $\operatorname{spec}^{*}(D)$ and the spectral partition of the volume.

Proof. This follows immediately from (5.26).

Finally, we note that the correspondence " $\lambda \leftrightarrow \frac{1}{\lambda}$ " interchanges the role of heat content asymptotics and Poisson spectrum. We can formalize this using a construction closely related to that of spectral zeta functions:

Definition 5.4. Suppose that $(G, \mathcal{O}, W)$ is a graph with geometry and that $D$ is a domain of $G$ with nonempty boundary. Let $\operatorname{spec}(D)$ be the Dirichlet spectrum associated to $D$ and let $a_{j}$ be defined as in (5.2). Let $s$ be a complex variable. The weighted zeta function associated to $D$ is defined to be

$$
\zeta_{D}(s)=\sum_{j} a_{j}^{2}\left(-\frac{1}{\lambda_{j}}\right)^{s} .
$$

We note that while $a_{j}$ depends on the choice of basis for each eigenspace, the definition of $\zeta_{D}(s)$ does not.

Corollary 5.4. Suppose that $(G, \mathcal{O}, W)$ is a graph with geometry and that $D$ is a domain of $G$ with nonempty boundary. Let $\zeta_{D}(s)$ be the zeta function associated to $D$ as in (5.30). Then, with $n$ a positive integer, with $A_{2, n}$ as in (1.6), and with $q_{n}$ as in (1.17),

$$
\begin{aligned}
\zeta_{D}(n) & =\frac{A_{2, n}}{n !}, \\
\zeta_{D}(-n) & =(-1)^{n} q_{n} n ! .
\end{aligned}
$$

Proof. The identity (5.31) follows from (5.5) and the definition of the zeta function. Similarly, the identity (5.32) follows from (5.26).

\section{REFERENCES}

[A] D. Aldous, Applications of random walks on finite graphs, Selected Proceedings of the Sheffield Symposium on Applied Probability (Sheffield, 1989) IMS Lecture Notes Monograph Ser., 18, Inst. Math. Statist., Hayward, CA (1991), 12-26. MR 93g:60145

[B] N. Biggs, Algebraic potential theory on graphs, Bull. London Math. Soc. 29 (1998), 641682. MR 98m:05120

[BG] M. van den Berg and P. Gilkey, Heat content asymptotics of a Riemannian manifold with boundary, J. Funct. Anal., 120 (1994), 48-71. MR 94m:58215

[C] F. R. K. Chung, Spectral Graph Theory, Amer. Math. Soc. CBMS Regional Conference Series in Mathematics 92, Providence, RI, 1997, pp. 12-26. MR 97k:58183 
[DS] P. Diaconis and D. Stroock, Geometric bounds for the eigenvalues of Markov chains, Ann. Applied Prob. 1 (1991), 36-61. MR 92h:60103

[Do] J. Dodziuk, Difference equations, isoperimetric inequality and transience of certain random walks, Trans AMS 284 (1984), 787-794. MR 85m:58185

[DSn] P. G. Doyle and J. L. Snell, Random walks and electrical networks, MAA Carus Monographs 22, Washington, D.C., 1984. MR 89a:94023

[Du] R. Duffin, Discrete potential theory, Duke Math. J. 20 (1953), 233-251. MR 16:1119d

[F1] R. Forman, Difference operators, covering spaces and determinants, Topology 28 (1989), 413-438. MR 91a:57012

[F2] R. Forman, Determinants and Laplacians on graphs, Topology 32 (1993), 35-46. MR 94g:58247

[Ge] P. Gerl, Random walks on graphs with a strong isoperimetric inequality, J. Theor. Prob. 1 (1988), 171-188. MR 89g:60216

$[\mathrm{H}] \quad$ R. Has'minskii, Probabilistic representations of the solutions of some differential equations, In: Proc. 6th All-Union Conf. On Theor. Prob. and Math. Stat. (Vilnius 1960) (1960). (Russian)

[K] G. Kirchhoff, Über die Auflösung der Gleichungen auf Welche man beider Untersuchen der linearen Vertheilung galvanischer Ströme gefüft wird, Annalen der Physik und Chemie 72 (1847), 495-508.

[KM] K. K. J. Kinateder and P. McDonald, Variational principles for average exit time moments for diffusions in Euclidean space, Proc. Amer. Math. Soc. 127 (1999), 2767-2772. MR 99m:60121

$[\mathrm{KMM}]$ K. K. J. Kinateder, P. McDonald and D. Miller, Exit time moments, boundary value problems, and the geometry of domains in Euclidean space, Probab. Theory Related Fields 111 (1998), 469-487. MR 99h:60151

[M] P. McDonald, Isoperimetric conditions, Poisson problems and diffusions in Riemannian manifolds, Potential Analysis 16 (2002), 115-138.

[SH] J. A. Shohat and J. D. Tamarkin, The Problem of Moments, Amer. Math. Soc., New York, 1943. MR 5:5c

[Sp] F. Spitzer, Principles of Random Walk, Springer Verlag, New York, NY, 1976. MR 53:9383

[V1] N. Varopoulos, Isoperimetric inequalities and Markov chains, J. Funct. Anal. 63 (1985), 215-239. MR 87e:60124

[V2] N. Varopoulos, Brownian motion and random walks on manifolds, Ann. Inst. Fourier 34 (1984), 243-269. MR 85m:58186

[Z] D. Zwillinger et al., eds., CRC Standard Mathematical Tables and Formulae, 30th Edition, CRC, New York, 1996.

New College of Florida, 5700 N. Tamiani Trail, Sarasota, Florida 34243

E-mail address: ptm@virtu.sar.usf.edu

The Courant Institute of Mathematical Sciences, 251 Mercer Street, New York, NY 10012-1110

E-mail address: meyersr@cims.nyu.edu 